\title{
A Comparison of Portfolio Theory and Weighted Utility Models of Risky Decision Making
}

\author{
Paul E. Lehner \\ University of Michigan
}

\begin{abstract}
Weighted utility models attempt to account for risk preferences in terms of an expectation-like equation applied to the subjective evaluation of probabilities and outcomes, while Portfolio theory assumes that risk preference is a function of expected value and perceived risk. A pair of empirical predictions which contrasts Portfolio theory with all weighted utility models is derived and tested. Results indicate that both theories are in error. In the case of Portfolio theory, it is clearly the axiom of single-peakedness over risk that is violated.
\end{abstract}

A number of theories of decision making under risk belong to the general category of weighted utility models (Anderson \& Shanteau, 1970; Edwards, 1955, 1962; Karmarkar, 1978; Kahneman \& Tversky, 1979). If we let gamble $A$ be denoted by $\left(x_{1}, p_{1} ; x_{2}, p_{2} ; \ldots ; x_{n}, p_{n}\right)$ where $p_{1}, p_{2}$, $\ldots, p_{n}$ are the probabilities of obtaining outcomes $x_{1}, x_{2}, \ldots, x_{n}$, then according to the weighted utility principle, the weighted utility of this gamble is

$$
\begin{aligned}
W U(\mathrm{~A}) & =\left[w\left(p_{1}\right) V\left(x_{1}\right)+w\left(p_{2}\right) V\left(x_{2}\right)+\ldots\right. \\
& \left.+w\left(p_{n}\right) V\left(x_{n}\right)\right] / W\left(p_{1}, p_{2}, \ldots, p_{n}\right),
\end{aligned}
$$

where $w$ and $V$ are function on the probabilities and outcomes, respectively, and $W$ is a function on the probability distribution. Furthermore, if we have two risky options, $A$ and $B$, then,

$$
A>{ }_{\mathrm{p}} B \quad \text { if and only if } W U(\mathrm{~A})>W U(\mathrm{~B}),
$$

where $>_{p}$ represents a weak order on preference. In most cases the function $W$ is a constant.

One theory which does not fall into this category is Portfolio Theory (Coombs, 1969, 1975; van Santen, 1978). According to Portfolio Theory, the preference order on a set of gambles is mediated by two variables; (1) the expected value $(E V)$ and (2) the perceived riskiness of each of the gambles. Furthermore, whenever a set of gambles are equal in $E V$, then the preference order on these gambles is single-peaked over the risk order. That is, if we have three gambles, $A, B$, and $C$ for which

$$
E V(A)=E V(B)=E V(C),
$$

Send reprint requests to: Paul E. Lehner, Mathematical Psychology Program, Department of Psychology, University of Michigan, Ann Arbor, MI 48109. 
and

$$
A>_{\mathrm{r}} B>_{\mathrm{r}} C \text {, }
$$

where $>_{\mathrm{r}}$ denotes a weak order or risk, then,

$$
\mathrm{B}>_{\mathrm{p}} \mathrm{A} \quad \text { or } \mathrm{B}>_{\mathrm{p}} \mathrm{C} \text {, }
$$

or both.

The concept of risk, however, is left undefined. In order to test Portfolio Theory, therefore, it is necessary to make some ad hoc assumptions about risk. When such assumptions are made, however, Portfolio Theory usually does an excellent job of predicting experimental results (Coombs, Donnell, \& Kirk, 1978; Coombs \& Huang, 1970, 1962). Particularly impressive are the results in Coombs et al. (1976) where singlepeakedness over risk was satisfied even though some of the stimuli were complex, viz., four outcome lotteries. To date, there is no published evidence showing any consistent violations of the single-peakedness axiom.

In this paper a pair of contrasting properties of weighted utility models and Portfolio Theory is derived and experimentally tested.

Consider the set of all three outcome gambles of the form

$$
(X+K, P ;-X+K, P ; K, 1-2 P) .
$$

It is obvious that any gamble of this form in uniquely defined by its expected value $(K)$, range $(2 X)$ and the symmetric probability of winning or losing $(P)$. Therefore, any gamble of this form can be rewritten as

$$
(K, R, P) \text {, }
$$

where $K$ is the expected value of the gamble, $R$ is the range, and $P$ is the symmetric probability. For example, the gamble

$$
(\$ 1, .25 ;-\$ 1, .25 ; \$ 0, .5)
$$

can be rewritten as

$$
(\$ 0, \$ 2, .25) \text {. }
$$

In order to examine what Portfolio Theory says about the preference order on this set of gambles, we must first have some knowledge about the risk order. Fortunately, much of the risk order on this set of gambles is obvious. To begin with, risk increases as $R$ increases. That is, if we have two gambles, $A$ and $A^{\prime}$, which are identical in every way except that the range of $A$ is greater than the range of $A^{\prime}$, it is intuitively compelling that $A$ is riskier than $A^{\prime}$. Similarly, it is also intuitively compelling to assume that risk increases as $P$, the symmetric probability, increases. By increasing $P$, we have in effect increased the variance by making it more likely that one of the two extreme outcomes will occur. 
Both of these assumptions about risk have been supported experimentally. Coombs and Bowen (1971a, 1971b), Coombs and Huang (1970), and Huang (1971) all found that, when $E V$ was held constant, risk judgments increased monotonically with increasing variance and range. In addition, as a result of an experiment studying preferences Coombs and Huang (1976; see also Coombs, 1976) also concluded that, when $E V$ was held constant, risk was monotone with $P$. In all of these experiments, several different $E V$ levels were used.

Given these assumptions about risk in this set of gambles, it is shown in the Appendix that for a preference order on a finite set of gambles with constant $E V$, the following three independent properties are necessary and sufficient conditions for there to exist a risk order, $\geqslant_{r}$, on the gambles such that,

(i) the preference order is single-peaked over the risk order, and

(ii) the risk order is strictly monotone with increasing $P$ and $R$.

Property 1 . Given any three gambles of the form

$$
(K, R, P), \quad\left(K, R^{\prime}, P\right), \quad\left(K, R^{\prime \prime}, P\right),
$$

where $R>R^{\prime}>R^{\prime \prime}$, then

$$
\left(K, R^{\prime}, P\right)>_{\mathrm{p}}(K, R, P)
$$

or

$$
\left(K, R^{\prime}, P\right)>_{\mathrm{p}}\left(K, R^{\prime \prime}, P\right) .
$$

Property 2. Given any three gambles of the form

$$
(K, R, P), \quad\left(\mathrm{K}, \mathrm{R}, \mathrm{P}^{\prime}\right), \quad\left(K, R, P^{\prime \prime}\right),
$$

where $P>P^{\prime}>P^{\prime \prime}$, then

$$
\left(K, R, P^{\prime}\right)>_{\mathrm{p}}(K, R, P)
$$

or

$$
\left(K, R, P^{\prime}\right)>_{\mathrm{p}}\left(K, R, P^{\prime \prime}\right) .
$$

Properties 1 and 2 follow directly from the definition of single peakedness and say in effect that preference is single peaked over range and symmetric probability.

Property 3. Given any four gambles of the form

$$
(K, R, P), \quad\left(K, R^{\prime}, P\right), \quad\left(K, R, P^{\prime}\right), \quad\left(K, R^{\prime}, P^{\prime}\right),
$$

where $R>R^{\prime}$ and $P>P^{\prime}$, then,

$$
\left(K, R^{\prime}, P\right) \text { and }\left(K, R, P^{\prime}\right)>_{\mathrm{p}}(K, R, P)
$$

or

$$
\left(K, R^{\prime}, P\right) \text { and }\left(K, R, P^{\prime}\right)>_{\mathrm{p}}\left(K, R^{\prime}, P^{\prime}\right) \text {. }
$$


Portofolio Theory, therefore, implies that properties 1 to 3 will be satisfied whenever the expected value is held constant.

A useful prediction that can be derived from property 3 is that, when an intermediate risk level is preferred, the preference orders on $P$ and $R$ cannot be mutually independent. This result is derived as follows. Let $A$, $B, C$, and $D$ be symmetric gambles with $P>P^{\prime}, R>R^{\prime}$, and $K$ a constant.

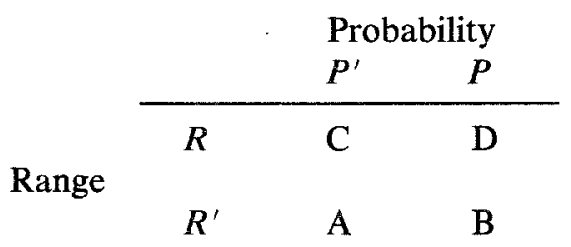

Given the previous assumptions about risk, we have $D>_{\mathrm{r}} B, C>_{\mathrm{r}} A$; and by single peakedness over risk we get

$$
B \text { and } C>{ }_{\mathrm{p}} A
$$

or

$$
B \text { and } C>{ }_{\mathrm{p}} D \text {, }
$$

which is the same as property 3 .

The definition of mutual independence however requires that,

$$
A>{ }_{\mathrm{p}} C \quad \text { if and only if } \quad B>_{\mathrm{p}} D
$$

and

$$
A>_{\mathrm{p}} B \quad \text { if and only if } \quad C>_{\mathrm{p}} D .
$$

Suppose $B$ is preferred to both $A$ and $D$, then mutual independence of probability and range requires that $A$ and $D$ be preferred to $C$, which violates (1). Similarly, if $C$ is preferred to both $A$ and $D$, then mutual independence requires that both $A$ and $D$ be preferred to $B$, which also violates (1). In this example, note that if $B$ or $C$ is preferred to both $A$ and $D$, and

$$
B \text { and } C>{ }_{\mathrm{p}} A \text {, }
$$

then we have an instance in which the most preferred range size decreases from $R$ to $R^{\prime}$ when the probability increases from $P^{\prime}$ to $P$. Similarly, if we have

$$
B \text { and } C>{ }_{\mathrm{p}} D \text {, }
$$

then we have a case in which the most preferred probability decreases from $P$ to $P^{\prime}$ when the range increases. Property 3 of the Portfolio Theory therefore predicts that, given the appropriate conditions, increasing the 
symmetric probability will lead people to prefer a smaller range; while increasing the range will lead people to prefer smaller probabilities.

Weighted utility models, on the other hand, disagree with property 3 . In particular, all weighted utility models predict the following property.

Property 4. For all $K$ fixed, the preference order over $R$ is independent of the preference order over $P$. That is,

$$
(K, R, P)>_{\mathrm{p}}\left(K, R^{\prime}, P\right),
$$

if and only if

$$
\left(K, R, P^{\prime}\right)>_{\mathrm{p}}\left(K, R^{\prime}, P^{\prime}\right) .
$$

This property is derived as follows. By the weighted utility principle,

$$
(K, R, P)>_{\mathrm{p}}\left(K, R^{\prime}, P\right) \text {, }
$$

if and only if

$$
\begin{aligned}
w(P) V[K+(R / 2)] & +w(P) V[K-(R / 2)]+w(1-2 P) V(K) \\
& >w(P) V\left[K+\left(R^{\prime} / 2\right)\right]+w(\bar{P}) V\left[K-\left(R^{\prime} / 2\right)\right] \\
& +w(1-2 P) V(K),
\end{aligned}
$$

which is true if and only if

$$
V[K+(R / 2)]+V[K-(R / 2)]>V\left[K+\left(R^{\prime} / 2\right)\right]+V\left[K-\left(R^{\prime} / 2\right)\right] .
$$

Since this last inequality does not include $P$, it is immediate that the preference order over $R$, with $P$ held constant, is independent of the choice of $P$.

Note that the derivation of property 4 relies primarily on the assumption that the evaluation of the probabilities is independent of the outcome each probability is associated with.

Weighted utility models therefore predict that preference for range is independent of the symmetric probability, while Portfolio Theory predicts that increasing the probability will, in certain cases, lead to preference for a smaller range.

The following experiment tests these four properties.

\section{METHOD}

\section{Subjects}

The subjects were 20 student volunteers from the University of Michigan paid subject pool.

\section{Stimuli}

The gambles in this experiment were all of the $(K, R, P)$ form mentioned above, and were constructed as indicated in Table 1 . There were three sets 
TABLE 1

Gambles Used as Stimuli

\begin{tabular}{|c|c|c|c|c|c|}
\hline \multirow[b]{2}{*}{$P$} & \multicolumn{5}{|c|}{$\mathrm{R}$} \\
\hline & $\$ .50$ & $\$ 1$ & $\$ 2$ & $\$ 4$ & $\$ 8$ \\
\hline \multicolumn{6}{|c|}{ Set I $(E V=\$ 0)$} \\
\hline .5 & $(\$ 0, \$ .50, .5)$ & $(\$ 0, \$ 1, .5)$ & $(\$ 0, \$ 2, .5)$ & $(\$ 0, \$ 4, .5)$ & $(\$ 0, \$ 8, .5)$ \\
\hline .4 & $(\$ 0, \$ .50, .4)$ & $(\$ 0, \$ 1, .4)$ & $(\$ 0, \$ 2, .4)$ & $(\$ 0, \$ 4, .4)$ & $(\$ 0, \$ 8, .4)$ \\
\hline .3 & $(\$ 0, \$ .50, .3)$ & $(\$ 0, \$ 1, .3)$ & $(\$ 0, \$ 2, .3)$ & $(\$ 0, \$ 4, .3)$ & $(\$ 0, \$ 8, .3)$ \\
\hline .2 & $(\$ 0, \$ .50, .2)$ & $(\$ 0, \$ 1, .2)$ & $(\$ 0, \$ 2, .2)$ & $(\$ 0, \$ 4, .2)$ & $(\$ 0, \$ 8, .2)$ \\
\hline .1 & $(\$ 0, \$ .50, .1)$ & $(\$ 0, \$ 1, .1)$ & $(0, \$ 2, .1)$ & $(\$ 0, \$ 4, .1)$ & $(\$ 0, \$ 8, .1)$ \\
\hline \multicolumn{6}{|c|}{ Set II $(E V=\$ 2)$} \\
\hline .5 & & & $(\$ 2, \$ 2, .5)$ & $(\$ 2, \$ 4, .5)$ & $(\$ 2, \$ 8, .5)$ \\
\hline .4 & & & $(\$ 2, \$ 2, .4)$ & $(\$ 2, \$ 4, .4)$ & $(\$ 2, \$ 8, .4)$ \\
\hline .3 & & & $(\$ 2, \$ 2, .3)$ & $(\$ 2, \$ 4, .3)$ & $(\$ 2, \$ 8, .3)$ \\
\hline .2 & & & $(\$ 2, \$ 2, .2)$ & $(\$ 2, \$ 4, .2)$ & $(\$ 2, \$ 8, .2)$ \\
\hline .1 & & & $(\$ 2, \$ 2, .1)$ & $(\$ 2, \$ 4, .1)$ & $(\$ 2, \$ 8, .1)$ \\
\hline \multicolumn{6}{|c|}{ Set III $(E V=\$ 5)$} \\
\hline .5 & & & $(\$ 5, \$ 2, .5)$ & $(\$ 5, \$ 4, .5)$ & $(\$ 5, \$ 8, .5)$ \\
\hline .4 & & & $(\$ 5, \$ 2, .4)$ & $(\$ 5, \$ 4, .4)$ & $(\$ 5, \$ 8, .4)$ \\
\hline .3 & & & $(\$ 5, \$ 2, .3)$ & $(\$ 5, \$ 4, .3)$ & $(\$ 5, \$ 8,3)$ \\
\hline .2 & & & $(\$ 5, \$ 2, .2)$ & $(\$ 5, \$ 4, .2)$ & $(\$ 5, \$ 8,2)$ \\
\hline .1 & & & $(\$ 5, \$ 2, .1)$ & $(\$ 5, \$ 4, .1)$ & $(\$ 5, \$ 8, .1)$ \\
\hline
\end{tabular}

of gambles. The 25 gambles in Set I are all at $E V=\$ 0$. The 15 gambles in Sets II and III are at $E V=\$ 2$ and $E V=\$ 5$, respectively.

Out of Sets I, II, and III a total of 47 subsets of gambles were constructed. Thirty-one of these subsets were taken from Set I. The five rows and five columns of Set $I$ in Table 1 make up 10 of these 31 subsets. An additional 16 consisted of those $2 \times 2$ submatrices from Set $I$ in Table 1 which had two adjacent levels of range and two adjacent levels of probability. For example, the set $(\$ 0, \$ .50, .1),(\$ 0, \$ .50, .2),(\$ 0, \$ 1.00, .1),(\$ 0$, $\$ 1.00, .2)$ was one of these subsets. Five additional subsets, one of size 5 and the rest of size 4 , were constructed from gambles of extreme range ( $R$ $=\$ .50$ or $\$ 8.00)$ or extreme probability $(P=.1$ or .5$)$. These last 5 subsets were added to guarantee that the subjects would be presented an equal number of pair comparison replications on all pairs of gambles that correspond to adjacent cells in Set $I$.

Eight subsets were constructed from Set II. They correspond to the eight rows and columns in the second matrix in Table 1 . In a similar manner, eight subsets were constructed from Set III.

All gambles were represented by a picture of a spinner board without the spinner. Both the winning and losing amounts and the probabilities were 
stated numerically. Furthermore, the probabilities were also represented by appropriate sections on the spinner board. The 47 subsets of gambles were presented to the subjects in the form of 47 small stacks of cards. On each card there was a picture representation of one gamble.

\section{Procedure}

Each subject participated in one experimental session, which lasted approximately $1 \mathrm{hr}$. After being familiarized with the gambles, subjects were asked to rank order the gambles in each subset with respect to preference. They were instructed to do this under the assumption that they would be playing only one gamble once.

The order of the gambles in each subset and the order in which the subsets were presented to each subject was randomized.

\section{RESULTS}

The first property of Portofolio Theory is that preference is single peaked over range. Table 2 gives the number of monotone, strictly folded (i.e., single peaked but not monotone), and non-single-peaked orders that subjects produced when they rank ordered sets of gambles with constant probability. Note that a monotone order is a special type of single-peaked order, so both monotone and strictly folded orders are consistent with Portfolio Theory.

Table 2 indicates that $90 \%$ of the 300 preference orders are single peaked. Since, by chance, we would expect only $46 \%$ of the orders to be single peaked, this result strongly supports property 1 of Portfolio Theory.

The second property of Portfolio Theory is that preference is single peaked over the symmetric probability. Table 3 gives the number of

TABLE 2

Classification of Preference Orders over

Range with Probability Held Constant

\begin{tabular}{lccc}
\hline & $\begin{array}{c}\text { Monotone } \\
\text { orders }\end{array}$ & $\begin{array}{c}\text { Strictly folded } \\
\text { orders }\end{array}$ & $\begin{array}{c}\text { Non-single-peaked } \\
\text { orders }\end{array}$ \\
\hline$E V=\$ 0$ & 74 & 21 & 5 \\
$E V=\$ 2$ & 56 & 32 & 12 \\
$E V=\$ 5$ & 71 & 16 & 13 \\
Total & $201(67 \%)$ & $69(23 \%)$ & $30(10 \%)$ \\
$\begin{array}{l}\text { Number expected } \\
\text { by chance }\end{array}$ & 68.3 & & \\
\hline
\end{tabular}


TABLE 3

Classification of Preference Orders over

Probability with Range Held Constant

\begin{tabular}{lccc}
\hline & $\begin{array}{c}\text { Monotone } \\
\text { orders }\end{array}$ & $\begin{array}{c}\text { Strictly folded } \\
\text { orders }\end{array}$ & $\begin{array}{c}\text { Non-single-peaked } \\
\text { orders }\end{array}$ \\
\hline$E V=\$ 0$ & 43 & 30 & 17 \\
$E V=\$ 2$ & 24 & 22 & 14 \\
$E V=\$ 5$ & 25 & 18 & 17 \\
Total & $102(46 \%)$ & $70(32 \%)$ & $48(22 \%)$ \\
$\begin{array}{l}\text { Number expected } \\
\text { by chance }\end{array}$ & 3.7 & & \\
\hline
\end{tabular}

monotone, strictly folded, and nonpeaked orders that subjects produced when they rank ordered sets of gambles with range held constant.

Table 3 indicates that $78 \%$ of the 220 preference orders are single peaked. By chance, we would have expected only $13 \%$ to be single peaked. Therefore, property 2 of Portfolio Theory is also verified.

Subjects rank ordered 16 subsets of four gambles each in which both range and probability were varied. For each of these subsets, there were 24 possible rank orders. Of the 24 possible rank orders, 12 satisfy the weighted utility property of independence of range with probability. Six of these 12 orders are consistent with property 3 of Portfolio Theory, while the remaining 6 orders violate Portfolio Theory. For the 12 orders that violate independence of range with probability, 6 show a smaller range preference with increasing probability, and 6 show a greater range preference with increasing probability. Therefore, of the 12 orders which violate independence of range with probability, 6 satisfy and 6 violate Portfolio Theory. Table 4 gives the observed number of rank orders in each of these categories.

TABLE 4

Classification of Preference Orders for the Sixteen $2 \times 2$ Submatrices

\begin{tabular}{lcc}
\hline & $\begin{array}{c}\text { Satisfy } \\
\text { Portfolio Theory }\end{array}$ & $\begin{array}{c}\text { Violate } \\
\text { Portfolio Theory }\end{array}$ \\
\hline $\begin{array}{l}\text { Satisfy } \\
\text { weighted utility } \\
\begin{array}{l}\text { Violate } \\
\text { weighted utility }\end{array}\end{array}$ & 216 & $64^{b}$ \\
\hline
\end{tabular}

${ }^{a}$ The probability of obtaining a ratio of $25: 15$ in an equal probability binomial distribution is less than .08. In future tables this will be marked as $p(25: 15)<.08$ (one-tailed test).

${ }^{b} p(64: 25)<.0001$ (one-tailed test). 
The data in Table 4 indicate that the weighted utility property of independence of range with probability does a better job of accounting for the preference orders than Portfolio Theory $(p>.001)$. However, for those preference orders which do violate property 4 , the majority satisfy Portfolio Theory. Though the size of the effect is small $(p<.08)$, this last result suggests that the property of independence of range with probability is violated in a manner consistent with Portfolio Theory; that is, increasing the symmetric probability leads to preference for a smaller range.

In order to examine this prediction of decreasing range preference with increasing probability more thoroughly, we reexamined the preference orders in the following manner. At each $E V$ level, we compared the most preferred level of range at the smallest level of probability for which preference was single-peaked over range, with the most preferred level of range at the highest probability level for which range preference was single-peaked. In this way, we could examine the changes in range preference as we went from low to high probability. According to property 3 of Portfolio Theory, we would expect preference orders at each $E V$ level to be either (1) consistently monotone over range, in which case independence of range from probability will be satisfied, or (2) sometimes strictly folded over range, in which case, when the probability increased, there should be a trend toward decreasing range preference. Weighted utility models, on the other hand, predict that independence of range with probability will always be satisfied. Table 5 gives the number of subjects for whom range preference increased, decreased, or stayed the same as the symmetric probability increased. The data in Table 5 clearly indicates a trend toward decreasing range preference. In particular, we note that there was only one case of increasing range preference $(p<.003)$. How-

TABLE 5

Changes in Range Preference with Increasing Probability

\begin{tabular}{|c|c|c|c|c|c|}
\hline & \multirow[b]{2}{*}{$\begin{array}{c}\text { Smaller } \\
\text { range }\end{array}$} & \multicolumn{2}{|c|}{ No change } & \multirow[b]{2}{*}{$\begin{array}{l}\text { Larger } \\
\text { range }\end{array}$} & \multirow[b]{3}{*}{$\begin{array}{c}\text { Cannot } \\
\text { test }\end{array}$} \\
\hline & & $\begin{array}{l}\text { Monotone } \\
\text { orders }\end{array}$ & $\begin{array}{l}\text { Folded } \\
\text { orders }\end{array}$ & & \\
\hline & $\begin{array}{c}\text { Satisfy PT } \\
\text { Violate WU }\end{array}$ & $\begin{array}{l}\text { Satisfy PT } \\
\text { Satisfy WU }\end{array}$ & $\begin{array}{l}\text { Violate PT } \\
\text { Satisfy WU }\end{array}$ & $\begin{array}{l}\text { Violate PT } \\
\text { Violate WU }\end{array}$ & \\
\hline$E V=0$ & 4 & 14 & 2 & 0 & 0 \\
\hline$E V=2$ & 4 & 9 & 5 & 1 & 1 \\
\hline$E V=5$ & 3 & 13 & 3 & 0 & 1 \\
\hline Total & $11^{a}$ & 36 & 10 & $1^{a}$ & 2 \\
\hline
\end{tabular}

${ }^{a} p(11: 1)<.003$ (one-tailed test). 
ever, it should also be noted that, in many instances, range preference remained unchanged.

Summarizing these last two results, we find that, as the probability increases, there is a trend toward decreasing range preference. However, this effect is not as strong and does not occur as often as Portfolio Theory would imply, but occurs more often than any weighted utility model could account for.

\section{DISCUSSION}

These results indicate that both Portfolio Theory and weighted utility models are inadequate theories of risky decision making.

In the case of Portfolio Theory, either the single-peakedness axiom or one of the risk assumptions must be incorrect. The fact that the risk assumptions are both intuitively reasonable and empirically well supported, therefore, suggests that it is the single-peakedness axiom that is in error. Furthermore, if the single-peakedness axiom were hypothesized to be correct, it would be difficult to explain how preference could be single peaked over the dimensions of range and symmetric probability (recall that properties 1 and 2 were satisfied), when risk does not increase monotonically with these dimensions.

In the case of the weighted utility models, it is the assumption that probabilities and outcomes are assessed independently that appears to be violated. This would suggest that risk preferences cannot be adequately represented by a model which proposes that preference judgments reflect a simple composition of outcomes and probabilities.

\section{APPENDIX: PROOF OF PROPERTIES 1, 2, AND 3}

The following proof of properties $1-3$ relies heavily on work found in Lehner (1980).

Let $G$ be a set of gambles of the $(K, R, P)$ form. Assume that all the gambles in $G$ have the same expected value. We begin with the following lemma.

\section{Lemma 1}

Properties 1-3 are satisfied if and only if, for any three distinct gambles,

$$
(K, R, P), \quad\left(K, R^{\prime}, P^{\prime}\right), \quad\left(K, R^{\prime \prime}, P^{\prime \prime}\right),
$$

with $R \geqslant R^{\prime} \geqslant R^{\prime \prime}$ and $P \geqslant P^{\prime} \geqslant P^{\prime \prime}$, we have

$$
\left(K, R^{\prime}, P^{\prime}\right)>_{\mathrm{p}}(K, R, P)
$$

or

$$
\left(K, R^{\prime}, P^{\prime}\right)>_{\mathrm{p}}\left(K, R^{\prime \prime}, P^{\prime \prime}\right) .
$$


Proof

Properties 1-3 are all special cases of (A1), consequently we need only show that properties 1-3 imply (A1).

Assume that we have at least two range levels $\left(R, R^{\prime}\right)$ and three probability levels $\left(P, P^{\prime}, P^{\prime \prime}\right)$. Assume also that there exist three gambles in $G$ which violate (A1), but do not violate properties 1-3.

In order to violate (A1) we must have three gambles for which

$$
(K, R, P) \geqslant_{\mathrm{p}}\left(K, R,{ }^{\prime}, P^{\prime}\right)
$$

and

$$
\left(K, R^{\prime \prime}, P^{\prime \prime}\right) \geqslant_{\mathrm{p}}\left(K, R^{\prime}, P^{\prime}\right) .
$$

By property 3 we have

$$
\left(K, R^{\prime}, P\right)>_{\mathrm{p}}(K, R, P) \quad \text { or } \quad\left(K, R^{\prime}, P^{\prime}\right)
$$

and

$$
\left(K, R^{\prime}, P^{\prime \prime}\right)>_{\mathrm{p}}\left(K, R^{\prime}, P^{\prime}\right) \quad \text { or } \quad\left(K, R^{\prime \prime}, P^{\prime \prime}\right) .
$$

Combining (A2) and (A3) gives us

$$
\left(K, R^{\prime}, P\right)>_{\mathrm{p}}\left(K, R^{\prime}, P^{\prime}\right) \text {. }
$$

Similarly, (A2) and (A4) together imply

$$
\left(K, R^{\prime}, P^{\prime \prime}\right)>_{\mathrm{p}}\left(K, R^{\prime}, P^{\prime}\right) .
$$

Finally, combining (A5) and (A6) gives us

$$
\left(K, R^{\prime}, P\right) \quad \text { and } \quad\left(K, R^{\prime}, P^{\prime \prime}\right)>_{\mathrm{p}}\left(K, R^{\prime}, P^{\prime}\right) .
$$

This last inequality violates property 2 , so we have a contradiction.

In cases where there are only two probability levels, but at least three range levels, a similar proof showing a violation of property 1 could be constructed. In cases where there are less than three levels of both range and symmetric probability no special proof is needed, since one of the three properties can be applied to any three-element subset.

\section{Lemma 2}

For the set of gambles $G$, a weak ordering, $\geqslant_{\mathrm{p}}$, is single peaked over some risk ordering, $\geqslant_{\mathrm{r}}$, that is strictly monotone with increasing range and probability if and only if (Al) is never violated.

Proof

See theorems 1 and 2 and lemma 6 in Lehner (1980).

Together, lemmas 1 and 2 immediately imply the following theorem. 


\section{Theorem 1}

A weak ordering, $\geqslant_{\mathrm{p}}$, is single peaked over some risk ordering, $\geqslant_{\mathrm{r}}$, that increases monotonically with range and probability if and only if properties $1-3$ are satisfied.

\section{REFERENCES}

Anderson, N. H., \& Shanteau, J. C. Information integration in risky decision making. Journal of Experimental Psychology, 1970, 84, 441-451.

Coombs, C. H. Portfolio Theory: A theory of risky decision making. La decsion. Paris: Centre National de la Rechereche Scientifique, 1969.

Coombs, C. H. Portfolio theory and the measurement of risk. In S. Schwartz and M. Kaplan (Eds.), Human judgement and decision processes. Academic Press, 1975. Pp. 63-85.

Coombs, C. H., \& Bowen, J. N. A test of $V E$-theories of risk and the effect of the central limit theorem. Acta Psychologica, 1971, 35, 15-28. (a)

Coombs, C. H., \& Bowen, J. N. Additivity of risk in portfolios. Perception and Psychophysics, 1971, 10, 43-46. (b)

Coombs, C. H., Donnel, M. L., \& Kirk, D. B. An experimental study of risk preferences in lotteries. Journal of Experimental Psychology: Human Perception and Performance, $1978,4(3), 492-512$.

Coombs, C. H., \& Huang, L. C. Tests of a portfolio theory of risk preference. Journal of Experimental Psychology, 1970, 85, 23-29.

Coombs, C. H., \& Huang, L. C. Polynomial psychophysics of risk. Journal of Mathematical Psychology, 1970, 7, 317-338.

Coombs, C. H., \& Huang, L. C. Tests of the betweeness property of expected utility theory. Journal of Mathematical Psychology, 1976, 13, 323-337.

Edwards, W. The prediction of decision making among bets. Journal of Experimental Psychology, 1955, 50, 201-214.

Edwards, W. Subjective probabilities inferred from decisions. Psychological Review, 1962, 62(2), 109-135.

Huang, L. C. Experiments on the measurement of risk. Michigan Mathematical Psychology Program Technical Report, (MMPP 71-7), the University of Michigan, 1971.

Karmarkar, U. S. Subjectively weighted utility: A descriptive extension of the expected utility model. Organizational Behavior and Human Performance, 1978, 21, 61-72.

Lehner, P. E. Folded additive structures: A nonpolynominal model of some factorial interactions. Michigan Mathematical Psychology Program Technical Report, (MMPP 80-1), the University of Michigan, 1980.

van Santen, J. P. H. A new axiomatization of Portfolio Theory. Journal of Mathematical Psychology, 1978, 17, 14-20.

RECEIVED: October 15, 1979 